distribution in liver and muscle cells, which can also influence insulin action.

Address correspondence to: Samuel Klein, Washington University School of Medicine, 660 South Euclid Avenue, Campus Box 8031, St. Louis, Missouri 63110, USA. Phone: (314) 362-8699; Fax: (314) 362-8230.

1. Vague, J. 1956. The degree of masculine differentiation of obesities: a factor determining predisposition to diabetes, atherosclerosis, gout, and uric calculous disease. Am. J. Clin. Nutr. 4:20-34.

2. Kissebah, A.H., et al. 1982. Relation of body fat distribution to metabolic complications of obesity. J. Clin. Endocrinol. Metab. 54:254-260.

3. Folsom, A.R., et al. 2000. Associations of general and abdominal obesity with multiple health outcomes in older women. Arch. Intern. Med. 160:2117-2128.

4. Chan, J.M., et al. 1994. Obesity, fat distribution, and weight gain as risk factors for clinical diabetes in men. Diabetes Care. 17:961-969.

5. Poulliot, M.-C., et al. 1992. Visceral obesity in men. Diabetes. 41:826-834.

6. Ruderman, N., Chisholm, D., Pi-Sunyer, X., and Schneider, S. 1998. The metabolically obese, normalweight individual revisited. Diabetes. 47:699-713.
7. Abate, N., Burns, D., Peshock, R.M., Stray-Gundersen, J., and Grundy, S.M. 1995. Relationship of generalized and regional adiposity to insulin sensitivity in men. J. Clin. Invest. 96:88-98.

8. Pouliot, M.C., et al. 1994. Waist circumference and abdominal sagital diameter: best simple anthropometric indexes of abdominal visceral adipose tissue accumulation and related cardiovascular risk in men and women. Am. J. Cardiol. 73:460-468.

9. National Institutes of Health. 1998. Clinical Guidelines on the Identification, Evaluation, and Treatment of Overweight and Obesity in Adults: the Evidence Report [erratum 1998, 6:464]. Obes. Res. 6(Suppl. 2):51S-209S

10. Frayn, K.N. 2000. Visceral fat and insulin resistance: causative or correlative? Br. J. Nutr. 83(Suppl. 1):S71-S77.

11. Boden, G. 1997. Role of fatty acids in the pathogenesis of insulin resistance and NIDDM. Diabetes. 46:3-10.

12. Horowitz, J.F., Coppack, S.C., Paramore, D., Cryer, P.E., and Klein, S. 1999. Effect of short-term fasting on lipid kinetics in lean and obese women. Am.J. Physiol. 276:E278-E284.

13. Jensen, M.D., Haymond, M.W., Rizza, R.A., Cryer, P.E., and Miles, J.M. 1989. Influence of body fat distribution on free-fatty acid metabolism in obesity. J. Clin. Invest. 80:1168-1173.

14. Klein, S., Luu, K., Gasic, S., and Green, A. 1996. Effect of weight loss on whole-body and cellular lipid metabolism in severely obese humans. Am.J. Physiol. 270:E739-E745

15. Bjorntorp, P. 1990. Portal adipose tissue as a generator of risk factors for cardiovascular disease and diabetes. Arteriosclerosis. 10:493-496.

16. Abate, N., et al. 1996. Relationship of generalized and regional adiposity to insulin sensitivity in men with NIDDM. Diabetes. 45:1684-1693.

17. Goodpaster, B.H., Thaete, F.L., Simoneau, J., and Kelley, D.E. 1997. Subcutaneous abdominal fat and thigh muscle composition predict insulin sensitivity independently of visceral fat. Diabetes. 46:1579-1585.

18. Banerji, M.A., Chaiken, R.L., Gordon, D., Kral, J.G., and Lebovitz, H.E. 1995. Does intra-abdominal adipose tissue in black men determine whether NIDDM is insulin-resistant or insulin-sensitive? Diabetes. 44:141-145.

19. Colberg, S., Simoneau, J.-A., Thaete, F.L., and Kelley, D.E. 1995. Skeletal muscle utilization of free fatty acids in women with visceral obesity. J. Clin. Invest. 95:1846-1853.

20. Nielsen, S., Guo, Z.K., Johnson, C.M., Hensrud, D.D., and Jensen, M.D. 2004. Splanchnic lipolysis in human obesity. J. Clin. Invest. 113:1582-1588. doi: $10.1172 / \mathrm{JCI} 200421047$

21. Basso, L.V., and Havel, R.J. 1970. Hepatic metabolism of free fatty acids in normal and diabetic dogs. J. Clin. Invest. 49:537-547.

\title{
Getting stents to go with the flow
}

\section{R. Wayne Alexander}

Department of Medicine, Division of Cardiology, Emory University School of Medicine, Atlanta, Georgia, USA.

\begin{abstract}
Implantation of expandable stents into stenotic arteries after percutaneous coronary intervention to relieve arterial narrowing has become a standard therapeutic tool. The improvement in vascular interventional technology, and especially stent technology, has, arguably, outstripped understanding of the biologic consequences of opening an obstructed artery. In the case of bifurcation stenoses, new evidence suggests that opening a stenotic subsidiary branch may create unfavorable hemodynamics in the stented main branch that can lead to in-stent restenosis (see the related article beginning on page 1607).
\end{abstract}

The branching arterial system has complex hemodynamics. Flow is laminar in straight segments away from the ostia of side branches or the flow dividers that form the origin of subsidiary vessels of a main branch. The inherently disturbed flow at branch points creates an environment that predisposes to the development of atherosclerosis $(1,2)$. This permissive environment is characterized by low, oscillating, or reversed flow created opposite flow dividers or branch points and is caused by flow separation in which streamlines of flowing blood curve away from the artery wall

Conflict of interest: The author has declared that no conflict of interest exists.

Citation for this article: J. Clin. Invest. 113:1532-1534 (2004). doi:10.1172/JCI200422000. proximally and back toward the wall distally (3). The impact of local hemodynamic influences on the pathogenesis of atherosclerosis has been studied extensively, both in vitro and in vivo (4). In vitro flow models have defined the effects of a given geometry on flow dynamics, and biologic responses have been associated with predicted flow patterns $(5,6)$. Generally, these models have not incorporated consideration of the evolving effects of the dynamic outward or inward remodeling of arteries that is associated with atherosclerosis (7). In particular, such approaches have not been applied extensively to exploration of the consequences of the acute hemodynamic changes inherent in the practice of interventional cardiology for clinical outcomes, specifically restenosis. The optimum approach to the clinical situation of, for example, the presence of proximal stenoses in both the main branch and a relatively large side branch of a coronary artery has not been agreed upon generally (8). With the advent of the use of vascular stents, a common approach has been to stent both arteries, although longterm outcomes are less than optimal (9). A guiding clinical principle has been the drive for complete revascularization. The underlying vascular biology and, in particular, the interdependence of the hemodynamic environment in the main and side branches in the presence of stenoses in each has been poorly understood. Thus, beyond the imperative of relieving ischemia, there has been no firm biologic basis guiding clinical decision-making with regard to intervention to open a severely stenotic side branch when percutaneous coronary intervention is being performed to open the main branch stenosis.

\section{In vitro simulation of flow patterns of branching arteries}

In this issue of the JCI, Richter and colleagues describe experimental approaches that represent a major advance in understanding the interrelatedness of stenoses 


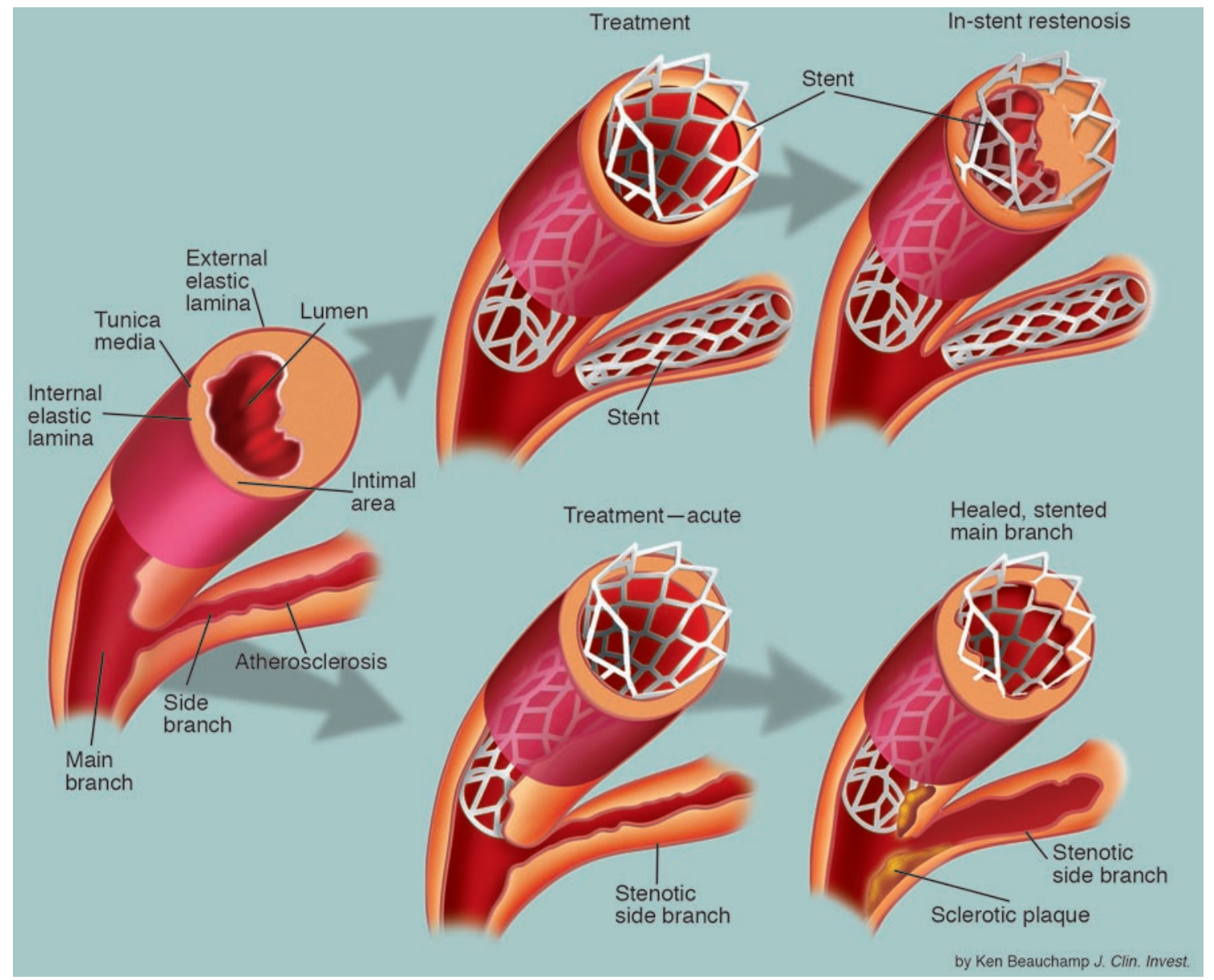

\section{Figure 1}

Potential influence of the status of a stenotic side branch on the healing of a main branch stenosis treated with percutaneous intervention and stent placement. Simultaneous stenting of the side branch may create disturbed flow patterns at the lateral wall opposite the side branch orifice, which are associated with amplified inflammatory responses and restenosis (upper panel). In the continued presence of stenosis and decreased flow in the side branch, the hemodynamic environment in the stented main branch may be similar to that in a nonbranching arterial segment with nondisturbed, laminar flow (lower panel). Laminar flow, in general, has anti-inflammatory and growth-inhibiting effects on the arterial wall and facilitates healing. While hypothetical, this figure illustrates that opening of a side branch in the setting of a stented main branch may have untoward effects on the outcome of the primary treatment target.

at branch points (10). They developed elegant in vitro models of a branching artery in which flow in both branches and the extent of stenosis in the side branch were controlled. Furthermore, they incorporated into the system compensatory remodeling in the main branch that would be stimulated by side branch occlusion. They evaluated the appearance of boundary layer separation under various circumstances. Arbitrary bifurcating geometries were associated with flow separation in both main and side branches. However, minor manipulations of the shape of the main branch lateral wall and of flow in both branches could readily be found that were associated with an absence of flow separation in the main branch and only a minimal amount of flow separation at the ostium of the patent side branch; these manipulations thus simulated a "healthy" hemodynamic state. A highgrade stenosis could be gradually induced in the side branch without the development of flow separation in the main branch as long as outward, compensatory remodeling in the main branch was incorporated into the system. Under these circumstances, the hemodynamic environment of the main branch was not different from that in a normal, nonbranching segment. Clinical circumstances were replicated in which the side branch stenosis was acutely removed in a setting in which compensatory outward main branch remodeling was not incorporated into the system. In this situation, opening the side branch was associated with significant flow separation along the main branch lateral wall. Thus, the hemodynamic environment in the main branch could be determined to a large extent by events and circumstances in the side branch. These observations led to predictions that the response to the injury of stent implantation in a main branch at an arterial bifurcation in their porcine model would be importantly influenced by the state of patency of the side branch, and by whether the main branch had been given sufficient time to remodel and adapt to prior side branch occlusion.

\section{Disturbed flow drives in-stent inflammation and lesion formation}

The most important finding of Richter and colleagues is that the in vivo results of side branch stenosis and main branch stenting were consistent with and predicted by the hemodynamic influences observed in the in 
vitro model (10) (Figure 1). Thus, in the presence of an occluded or stenotic side branch, stent implantation in the main branch was associated only with a concentric, uniform, cell-rich region, composed to a large extent of vascular smooth muscle cells around the stented portion of the artery. This resembled the vessel remodeling expected in a straight, nonbifurcating segment with nondisturbed flow. In contrast, dilating and stenting of the stenotic side branch and subsequent stenting of the main branch before it was allowed to remodel was associated with the development of an eccentric main branch, in-stent lesion that was located on the lateral wall beginning opposite the side branch orifice. This location was correlated with the area of flow separation in the in vitro model. On the other hand, permitting the main branch to remodel before stenting it and before opening and stenting the side branch mitigated the formation of the eccentric, in-stent restenosis. Outward remodeling of the main branch in the in vitro model before opening the side branch was associated with normalization of the flow patterns. Thus, disturbed, nonlaminar flow in vivo, as predicted from the in vitro model, was associated consistently with restenotic lesion formation in the stented area.

The mechanism or mechanisms by which disturbed flow patterns and flow separation induce in-stent lesion formation involve inflammation. Leukocyte accumulation is characteristic of the chronic inflammatory response elicited by arterial stent implantation and drives the resulting remodeling $(11,12)$. This inflammatory response was most intense and asymmetric in the side-branch patent model and, as in the case of the neointimal hyperplasia, was concentrated along the main branch lateral wall, beginning opposite the side branch orifice. In addition to the assymmetric intimal hyperplasia in this location and setting there was a striking, crescent- shaped area in the outer part of the lateral wall that was hypocellular and connective tissue-rich. Thus abnormal flow patterns, as inferred from the in vitro model, appear to determine in the stented artery the local intensity of the inflammatory response that in turn drives both the abnormal proliferative and fibrotic responses.

These results are provocative in their own right in demonstrating the central role of the hemodynamic environment in determining the remodeling response (restenosis) in the stented artery. They also raise a number of important questions. Conventional wisdom assigns an important role to the endothelium in remodeling of the arterial wall (13). In this setting the endothelium is presumably absent. If so, what cells are sensing the abnormal flow environment that results from boundary layer separation? Presumptively, the mononuclear cells may sense and respond to the disordered flow environment. If so, what are the mechanisms involved? Is the intimal mononuclear cell population responsible for the crescentic, hypocellular area of the lesion, and what are the mechanisms? Intimal injury is associated with a robust cellular response in the adventitia (14). This phenomenon is not addressed here. Could it also play a role? Could robust anti-inflammatory strategies obviate the maladaptive responses imposed by the presence of the patent side branch? Could stent design and technology be adapted to create an optimum hemodynamic environment?

Richter and colleagues (10) have advanced our understanding of the biologic issues involved in the application of percutaneous intervention and stent technology to the treatment of bifurcation lesions. Potential clinical application will require considerable further investigation.

Address correspondence to: R. Wayne Alexander, Department of Medicine, Division of
Cardiology, Emory University School of Medicine, 1364 Clifton Road NE; EUH, H-153, MS 1600/002/1AA, Atlanta, Georgia 30322, USA. Phone: (404) 727-1749; Fax: (404) 7273099; E-mail: ralexan@emory.edu.

1. Ku, D.N., Giddens, D.P., Zarins, C.K., and Glagov, S. 1985. Pulsatile flow and atherosclerosis in the human carotid bifurcation. Positive correlation between plaque location and low and oscillating shear stress. Arteriosclerosis. 5:293-302.

2. Zarins, C.K., et al. 1983. Carotid bifurcation atherosclerosis. Quantitative correlation of plaque localization with flow velocity profiles and wall shear stress. Circ. Res. 53:502-514.

3. Fox, J.A., and Hugh, A.E. 1966. Localization of atheroma: a theory based on boundary layer separation. Br. Heart. J. 28:388-399.

4. Gimbrone, M.A., Jr., et al. 1997. Hemodynamics, endothelial gene expression, and atherogenesis. Ann. N. Y. Acad. Sci. 811:1-10; discussion 10-11.

5. Chappell, D.C., Varner, S.E., Nerem, R.M., Medford, R.M., and Alexander, R.W. 1998. Oscillatory shear stress stimulates adhesion molecule expression in cultured human endothelium. Circ. Res. 82:532-539.

6. DeKeulenaer, G.W., et al. 1998. Oscillatory and steady laminar shear stress differentially affect human endothelial redox state: role of a superoxideproducing NADH oxidase. Circ. Res. 82:1094-1101.

7. Galis, Z.S., and Khatri, J.J. 2002. Matrix metalloproteinases in vascular remodeling and atherogenesis: the good, the bad, and the ugly. Circ. Res. 90:251-262.

8. Lefevre, T., et al. 2000. Stenting of bifurcation lesions: classification, treatments, and results. Catheter. Cardiovasc. Interv. 49:274-283.

9. Baim, D. 1996. Is bifurcation stenting the answer? Cathet. Cardiovasc. Diagn. 37:314-316.

10. Richter, Y., Groothuis, A., Seifert, P., and Edelman, E.R. 2004. Dynamic flow alterations dictate leukocyte adhesion and response to endovascular interventions. J. Clin. Invest. 113:1607-1614. doi:10.1172/JCI200421007.

11. Rogers, C., Edelman, E.R., and Simon, D.I. 1998. $\mathrm{A} \mathrm{mAb}$ to the beta2-leukocyte integrin Mac-1 (CD11b/CD18) reduces intimal thickening after angioplasty or stent implantation in rabbits. Proc. Natl. Acad. Sci. U. S. A. 95:10134-10139.

12. Simon, D.I., et al. 2000. Decreased neointimal formation in Mac-1(-/-) mice reveals a role for inflammation in vascular repair after angioplasty. J. Clin. Invest. 105:293-300.

13. Rudic, R.D., et al. 1998. Direct evidence for the importance of endothelium-derived nitric oxide in vascular remodeling. J. Clin. Invest. 101:731-736.

14. Okamoto, E., et al. 2001. Perivascular inflammation after balloon angioplasty of porcine coronary arteries. Circulation. 104:2228-2235. 